\title{
A spatial Markov model for the evolution of the joint distribution of groundwater age, arrival time, and velocity in heterogeneous media
}

\author{
Arash Massoudieh ${ }^{1}$, Marco Dentz $^{2}$, and Jamal Alikhani ${ }^{1}$ \\ ${ }^{1}$ Civil Engineering, The Catholic University of America, Washington, DC, USA. \\ ${ }^{2}$ Spanish National Research Council (IDAEA-CSIC), Barcelona, Spain
}




\section{Abstract}

The evolution of the joint distribution of groundwater age, velocity and arrival times based on a Markov model for the velocities of fluid particles in heterogeneous porous media has been quantified. An explicit evolution equation for the joint distribution of age, arrival time and particle velocity is derived, which is equivalent to a continuous time random walk for age, velocity and arrival time. The approach is fully parameterized by the correlation model and the distribution of groundwater flow velocities. The transition probability for subsequent particle velocities along streamlines is implemented by a Copula, which is an efficient method to generate a correlated velocity series with prescribed marginal distribution. We discuss different solution methods based on finite-differences and random walk particle tracking. The latter is based on continuous time random walks, whose transition times are obtained kinematically from the flow velocities. Specifically, we discuss a renormalization scheme to accelerate the particle tracking simulations based on the definition of aggregate particle transitions which at the same time renormalizing velocity correlation. The impact of velocity correlation and velocity distribution on the evolution of age at different distances from the inlet plane is also studied. At distances of the order of the correlation length, persistent particle velocities gives the same behavior as stochastic streamtube models. For velocity distributions which give rise to transition times with finite variance, the age distributions evolve towards an inverse Gaussian. For heavy-tailed weighting times, they evolve towards stable distribution as the distance from the inlet increases.

\section{Introduction}

The concept of groundwater age or travel time distribution has received great attention recently due to its ability to inform about the sustainability of groundwater resources or their vulnerability to release of contaminants at ground surface [Bethke and Johnson, 2008; Cornaton and Perrochet, 2006; Leray et al., 2012; Cook and Solomon, 1997; Weissmann et al., 2002; Leray et al., 2016]. Because there is no direct experimental method for determining groundwater age, it is often inferred using measured concentrations of anthropogenic or cosmogenic tracers with known atmospheric records and decay or production rates in the aquifer [Massoudieh et al., 2012; Solomon et al., 2010; Cook and Böhlke, 2000]. This is often done by presuming a form for the travel time distribution referred to as a lumped parameter model (LPM) and then estimating the parameters of such models by matching predicted and measured tracer concentrations either as point estimates [Solomon et al., 2010; 
Leray et al., 2012; Maloszewski and Zuber, 1993] or probabilistically [Massoudieh et al., 2012; Alikhani et al., 2016]. One problem with this approach however is that although it may provide information on travel time distributions at each sampling location, it does not take into consideration the spatial relations between the travel time distributions at multiple sites that may exist due to the regional flow patterns, and therefore is sometimes incapable of providing big-picture knowledge about the flow patterns in an aquifer system. Furthermore, by considering the spatial relations between age distributions at multiple sampling locations, the problem can become more constrained resulting in a reduction in the uncertainty due to over-parameterization of lumped parameter models. Evaluating the tracer data at multiple locations in a holistic way can potentially provide insights into the nature of the groundwater flow including mean flow velocities, heterogeneity-structure, and connectivity in the absence of a calibrated detailed groundwater model.

When forward modeling has been used to determine age distributions, it has been traditionally assumed that the spatial evolution of the travel time distribution at any given point is controlled by the simple advection-dispersion equation (ADE) [Woolfenden and Ginn, 2009; Ginn, 2000; Massoudieh and Ginn, 2011]. However, it is known from numerous tracer breakthrough tests that the actual travel time distributions in heterogeneous systems often present tails that are typically not captured by simple ADE-based models [Berkowitz et al., 2006], hence leading to the use of non-conventional transport operators such as multidomain approaches [Ginn et al., 2009] non-local formulations, anomalous diffusion, and continuous-time random walk (CTRW) [Berkowitz et al., 2006; Engdahl et al., 2012]. From several perspectives, it is desirable to connect the spatial evolution of age distribution to physical properties of an aquifer such as the distribution of hydraulic conductivity and the connectivity structure. Firstly, this allows predicting age distribution based on the physical aquifer properties, and secondly, by linking the spatial evolution of age distribution, it is possible to use the information from multiple tracers collected at various locations (e.g. wells) to infer aggregate statistics of aquifer properties affecting the evolution of age distribution.

In this paper, we derive an approach for the evolution of the joint distribution of age, arrival time and velocity of fluid particles based on the notion that particle velocity series in steady heterogeneous flow fields can be modeled as a Markov process in distance along streamlines [Le Borgne et al., 2008; Kang et al., 2015; Dentz et al., 2016], which is the basis of the CTRW approach to transport in heterogeneous porous and fractured media [Berkowitz et al., 2006]. The distribution of fluid particle velocities can be related to the Eulerian ve- 
locity distribtion [Dentz et al., 2016], which in turn can be related to the distribution of hydraulic conductivity [Cvetkovic et al., 2014; Edery et al., 2014; Tyukhova et al., 2016]. In order to model the transition probability of subsequent particle velocities, the concept of copulas [Nelsen, 2013] is used. Although copulas have been used in other areas of hydrology [Favre et al., 2004; Salvadori and De Michele, 2007] and in particular in geostatistical interpolation [Bárdossy and Li, 2008], their utility in expressing correlation in studying transport phenomena in heterogeneous porous media or fractured media has been much less studied [Haslauer et al., 2012]. Copulas provide a systematic way to generate correlated velocity series for pre-determined marginal distributions. Based on (continuous time) random walk formulations of particle motion in steady heterogeneous flow fields, the evolution equations for the joint densities of age arrival time and particle velocities is derived in Section 3. The solution of the derived transport model using finite differences as well as random walk particle tracking has been discussed. An alternative approach refereed to as renormalization scheme for the efficient simulation of the evolution of arrival times and groundwater age based on the proposed modeling approach has also been presented. The evolution of the distribution of groundwater age is discussed in the light of the correlation of groundwater velocities and different classes of velocity distributions in Section 4.

\section{Groundwater Age Distribution}

Here we focus on the joint PDF of arrival time $t(x)$ and groundwater age $a[t(x)]$ at a position $x$. We first recall the case of advective-diffusive transport with constant groundwater flow $v_{0}$ and dispersion coefficient $D$ before we consider the case of variable groundwater flow velocities in the next section.

Considering a fluid particle based the Lagrangian viewpoint, the well known governing equations for arrival time and groundwater age for homogenous media can be obtained. The position $x(t)$ and age $a(t)$ of a fluid particle evolve in time according to the Langevin equations

$$
\frac{d x(t)}{d t}=v_{0}+\sqrt{2 D} \xi(t), \quad \frac{d a(t)}{d t}=1,
$$

where $\xi(t)$ is a Gaussian white noise with 0 mean and unit variance. The initial conditions at time $t_{0}$ are $x\left(t=t_{0}\right)=x_{0}$ and $a\left(t=t_{0}\right)=a_{0}$. The time at which a particle arrives at a position $x$ is defined by

$$
t(x)=\min \{t \mid x(t) \geq x\}
$$


The particle age is given by

$$
a(x)=a_{0}+t(x)-t_{0}
$$

where $t(x=0)=t_{0}$ is the time at which the particle enters the aquifer at the age $a_{0}$. In the following, without loss of generality, we set $a_{0}=0$. The joint probability density function (PDF) of arrival time, age and velocity can be written in terms of the particle trajectories as

$$
p\left(t, a, v ; x \mid t_{0}\right)=\langle\delta[t-t(x)]\rangle \delta\left[a-\left(t-t_{0}\right)\right] \delta\left(v-v_{0}\right) .
$$

Due to homogeneity in time, the PDF is a function of the difference $t-t_{0}$ of arrival and injection time, that is, the travel time,

$$
p\left(t, a, v ; x \mid t_{0}\right)=p\left(t-t_{0}, a, v ; x\right)
$$

where $p(t, a, v ; x)$ is the joint PDF of travel time, age and velocity. Note that $v$ is constant and equal to $v_{0}$, and age $a$ is determinisically related to the arrival time through (3). Thus, the joint density can be written as

$$
p\left(t-t_{0}, a, v ; x\right)=p_{a}\left(t-t_{0}, a ; x\right) \delta\left(v-v_{0}\right)=p_{t}\left(t-t_{0} ; x\right) \delta\left[a-\left(t-t_{0}\right)\right] \delta\left(v-v_{0}\right)
$$

where $p_{a}(t, a ; x)$ is the joint density of travel time and age, and $p_{t}(t ; x)$ is the travel time density. The joint density $p_{a}(t, a ; x)$ satisfies the advection-diffusion equation

$$
\frac{\partial p_{a}(t, a ; x)}{\partial t}+\frac{\partial p_{a}(t, a ; x)}{\partial a}+v_{0} \frac{\partial p_{a}(t, a ; x)}{\partial x}-D \frac{\partial^{2} p_{a}(t, a ; x)}{\partial x^{2}}=0 .
$$

The boundary condition is $p_{a}(t, a ; x=0)=\delta\left(t-t_{0}\right) \delta(a)$ at the inlet boundary and 0 at infinity. Note that $p_{a}\left(t-t_{0}, a ; x\right)$ quantifies the joint PDF of arrival time and age of fluid particles that are injected at the time $t_{0}$. The PDF of age of fluid particles that have been injected over all times until time $t$ is given by

$$
\int_{-\infty}^{t} d t_{0} p_{a}\left(t-t_{0}, a ; x\right) \equiv \rho_{w}(a ; x) .
$$

Under these steady-state conditions the age distribution is independent of time and satifies

$$
\frac{\partial \rho_{w}(a ; x)}{\partial a}+v_{0} \frac{\partial \rho_{w}(a ; x)}{\partial x}-D \frac{\partial^{2} \rho_{w}(a ; x)}{\partial x^{2}}=0
$$

for the boundary condition $\rho_{w}(a ; x=0)=\delta(a)$. Its solution is given by the inverse Gaussian distribution as [Ginn et al., 2009]

$$
\rho_{w}(a ; x)=\frac{x \exp \left[-\frac{\left(x-v_{0} a\right)^{2}}{4 D a}\right]}{\sqrt{4 \pi D a^{3}}} .
$$

In the following, we generalize these concepts for heterogeneous media, which are characterized by velocity transitions along and between streamlines. 


\section{Age Evolution in Heterogeneous Velocity Fields}

Let's consider the movement of fluid particles in steady heterogeneous velocity fields. Particles may change their velocity as they move along a streamline and due to transitions between streamlines caused by diffusion. We take again a Lagrangian viewpoint. Here, we describe the evolution of particle position along the trajectory as a function of the advective travel distance $s$ [Cvetkovic et al., 1991, 1996; Dentz et al., 2016]

$$
\frac{d s(t)}{d t}=v[s(t)]
$$

where $v(s)$ is the particle velocity at $s$. Advective-diffusive particle motion may then be written as

$$
d x(s)=d s+\sqrt{\frac{2 D d s}{v(s)}} \eta(s), \quad d t(s)=\frac{d s}{v(s)}, \quad d a(s)=\frac{d s}{v(s)},
$$

where $x(s)$ is the position along the particle trajectory. It is approximately equal to the travel distance from the inlet boundary. The particle velocity $v(s)$ may change due to velocity transitions along and between streamlines. Its evolution in steady heterogeneous porous media flows can be modeled as a Markov process in $s$ [Le Borgne et al., 2008; Kang et al., 2011; Dentz et al., 2016]. Specifically, we employ the process

$$
v(s+d s)=\zeta(s) v(s)+[1-\zeta(s)] v[s \mid v(s)] .
$$

The velocities $v(s \mid v)$ are distributed according to $p_{v}(v \mid v)$, where $\epsilon$ is a characteristic persistence length scale, and $\zeta(s)$ is a binary random variable according to the Bernoulli distribution that can take values of 0 or 1 . This velocity process is assumed to be stationary and ergodic. The steady state velocity PDF is denoted by $p_{v}(v)$ and fulfills

$$
p_{v}(v)=\int_{0}^{\infty} d v^{\prime} p_{v}\left(v \mid v^{\prime}\right) p_{v}\left(v^{\prime}\right) .
$$

Note that $p_{v}(v)$ is the PDF of particle velocities sampled equidistantly in space along the trajectory. As discussed in Dentz et al. [2016], it is related to the flux-weighted Eulerian velocity PDF. The $\zeta(s)$ is further distributed according to

$$
p_{\zeta}(\zeta)=\exp (-d s / \epsilon) \delta(\zeta-1)+[1-\exp (-d s / \epsilon)] \delta(\zeta)
$$

where $\epsilon$ is a persistence length scale. This means, at each step velocities persist with probability $\exp (-d s / \epsilon)$. If velocities change, then the new velocity is drawn from the PDF $p_{v}\left(v \mid v^{\prime}\right)$ conditioned on the previous velocity $v^{\prime}$. The velocity transition PDF for this stationary Markov 


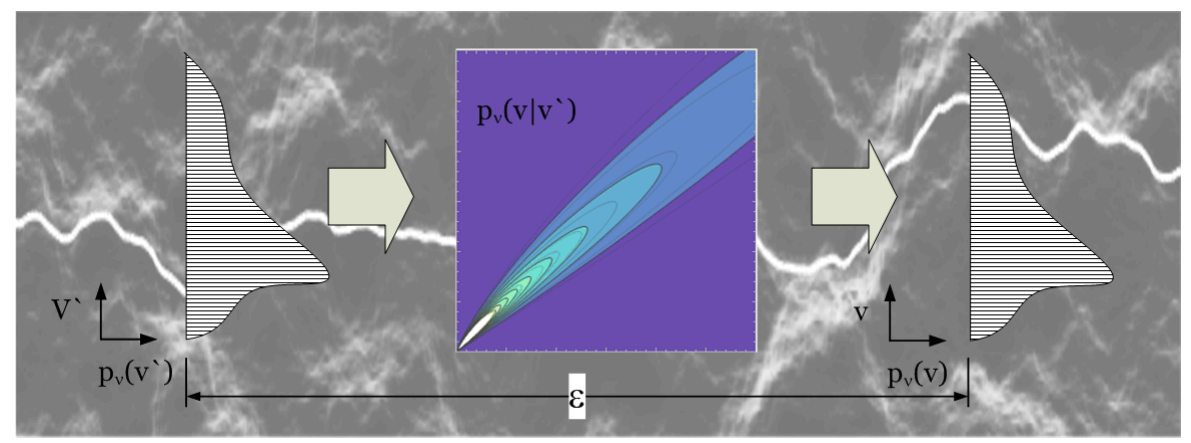

Figure 1. A schematic of the velocity transition conceptual model

process is given by [Dentz et al., 2016]

$$
r\left(v, d s \mid v^{\prime}\right)=\exp (-d s / \epsilon) \delta\left(v-v^{\prime}\right)+[1-\exp (-d s / \epsilon)] p_{v}\left(v \mid v^{\prime}\right)
$$

Figure 3 demonstrates the conceptual model for the velocity transition.

\subsection{Governing Equations}

The joint density of arrival time, age and particle velocity at a position $x$ is defined by

$$
p\left(t, a, v ; x \mid v_{0}, t_{0}\right)=\langle\delta[t-t(x)] \delta[v-v(x)]\rangle \delta\left[a-\left(t-t_{0}\right)\right]
$$

The boundary velocity and time are $v_{0}$ and $t_{0}$ such that

$$
p\left(t, a, v ; x=0 \mid v_{0}, t_{0}\right)=\delta\left(t-t_{0}\right) \delta\left(v-v_{0}\right) \delta\left[a-\left(t-t_{0}\right)\right] .
$$

Due to homogeneity in time, it is only a function of the travel time $t-t_{0}$,

$$
p\left(t, a, v ; x=0 \mid v_{0}, t_{0}\right) \equiv p\left(t-t_{0}, a, v ; x=0 \mid v_{0}\right) .
$$

It is the Green function of the transport problem. Note that the particle velocity is a random variable, while age is still deterministically related to travel time through (3). The joint density evolves according to (see Appendix A: )

$$
\begin{aligned}
& \left(\frac{\partial}{\partial a}+\frac{\partial}{\partial t}\right) p\left(t, a, v ; x \mid v_{0}\right)=-\left(v \frac{\partial}{\partial x}-D \frac{\partial^{2}}{\partial x^{2}}\right) p\left(t, a, v ; x \mid v_{0}\right) \\
& -\frac{v}{\epsilon} p\left(t, a, v ; x \mid v_{0}\right)+\frac{v}{\epsilon} \int d v^{\prime} p_{v}\left(v \mid v^{\prime}\right) p\left(t, a, v^{\prime} ; x \mid v_{0}\right)
\end{aligned}
$$

The change of the PDF over time and age is balanced through the advective-diffusive fluxes on the right side of the equation. Changes of velocity over time are represented by the last two terms on the right side. The first term denotes the probability per time for a transition 
from the present velocity to any other velocity. A particle encounters a new velocity with a spatial frequency $1 / \epsilon$, which translates into a temporal frequency through the present velocity as $v / \epsilon$. The second term is the probability per time of a velocity transition from any previous velocity $v^{\prime}$ to the present velocity $v$.

We now consider the joint PDF $\rho\left(t, a, v ; x, t_{0}\right)$, which satisfies (19) for the boundary condition

$$
\rho\left(t, a, v ; x=0, t_{0}\right)=\delta\left(t-t_{0}\right) p_{0}\left(v, t_{0}\right) \delta\left[a-\left(t-t_{0}\right)\right] .
$$

This means, the velocities of fluid particles that are injected at time $t_{0}$ have boundary velocities that are distributed according to of $p_{0}\left(v, t_{0}\right)$. The joint PDF $\rho\left(t, a, v ; x, t_{0}\right)$ is obtained from the Green function (16) as

$$
\rho\left(t, a, v ; x, t_{0}\right)=\int_{0}^{\infty} d v_{0} p\left(t-t_{0}, a, v ; x \mid v_{0}\right) p_{0}\left(v, t_{0}\right) .
$$

For a continuous injection over the boundary at $x=0$, we obtain the joint PDF $\rho_{a}(a, v ; x, t)$ of age and velocity from $\rho(t, a, v ; x, t)$ by integration over time as

$$
\rho_{a}(a, v ; x, t)=\int_{-\infty}^{t} d t_{0} p\left(t, a, v ; x, t_{0}\right) .
$$

It satisfies

$$
\begin{aligned}
& \left(\frac{\partial}{\partial a}+\frac{\partial}{\partial t}\right) \rho_{a}(a, v ; x, t)=-\left(v \frac{\partial}{\partial x}-D \frac{\partial^{2}}{\partial x^{2}}\right) \rho_{a}(a, v ; x, t) \\
& -\frac{v}{\epsilon} \rho_{a}(a, v ; x, t)+\frac{v}{\epsilon} \int d v^{\prime} p_{v}\left(v \mid v^{\prime}\right) \rho_{a}\left(a, v^{\prime} ; x, t\right) .
\end{aligned}
$$

for the boundary condition

$$
\rho(a, v ; x=0, t)=\delta(a) p_{0}(v, t) .
$$

It should be noted that an alternative equation for the evolution of residence age distribution with time and space can be obtained through the application of water balance (or Reynolds transport theorem) as shown in Appendix B: . Under the steady flow conditions under consideration here, the velocity PDF at the injection boundary is equal to the flux weighted Eulerian velocity $\mathrm{PDF}$, which, as mentioned above is equal to the steady state $\operatorname{PDF} p_{0}(v, t) \equiv$ $p_{\nu}(v)$ of the velocity Markov process (12b). Under these conditions the joint PDF of age and velocity is time-independent,

$$
\rho_{a}(a, v ; x, t) \equiv \rho_{a}(a, v ; x)
$$


It evolves according to

$$
\begin{aligned}
& \frac{\partial}{\partial a} \rho_{a}(a, v ; x)=-\left(v \frac{\partial}{\partial x}-D \frac{\partial^{2}}{\partial x^{2}}\right) \rho_{a}(a, v ; x) \\
& -\frac{v}{\epsilon} \rho_{a}(a, v ; x)+\frac{v}{\epsilon} \int d v^{\prime} p_{v}\left(v \mid v^{\prime}\right) \rho_{a}\left(a, v^{\prime} ; x\right) .
\end{aligned}
$$

for the boundary condition $\rho_{a}(a, v ; x=0)=\delta(a) p_{v}(v)$. The age distribution $\rho_{w}(a ; x)$ is obtained by marginalization of $\rho(a, v ; x)$ as

$$
\rho_{w}(a ; x)=\int_{0}^{\infty} d v \rho_{a}(a, v ; x) .
$$

It is instructive to consider the special case of perfectly correlated velocities. In this case, we set $p_{v}\left(v \mid v^{\prime}\right)=\delta\left(v-v^{\prime}\right)$. Equation (26) then becomes

$$
\frac{\partial}{\partial a} \rho_{a}(a, v ; x)=-\left(v \frac{\partial}{\partial x}-D \frac{\partial^{2}}{\partial x^{2}}\right) \rho_{a}(a, v ; x) \text {. }
$$

Its solution for the boundary condition $\rho_{a}(a, v ; x=0)=\delta(a) p_{v}(v)$ is given by

$$
\rho_{a}(a, v ; x)=p_{v}(v) \frac{x \exp \left[-\frac{(x-v a)^{2}}{4 D a}\right]}{\sqrt{4 \pi D a^{3}}} .
$$

The velocity within each particle trajectory is constant and equal to the velocity $v_{0}$ at the boundary. In this case, the correlated model reduces to the well-known stochastic convective streamtube approach [Dagan and Bressler, 1979], see also the discussion in Kang et al. [2015]. The age distribution $\rho_{w}(a ; x)$ is given by (27) and reduces in the limit $D \rightarrow 0$ to

$$
\rho_{w}(a ; x)=\frac{x}{a^{2}} p_{v}(x / a)
$$

Note that (19) provides an evolution equation for the joint distribution of age and velocity as a function of distance from the inlet and time. Specifically, the distribution of age here is equivalent to the distribution of travel times from the inlet boundary to the sampling position $x$. In Appendix B: the derivation of the equation for the joint distribution of age and velocity within a control volume located at position $x$ is presented.

\subsection{Velocity copula}

The conditional PDF $p_{v}\left(v \mid v^{\prime}\right)$ of velocity is represented through the copula $c\left(u, u^{\prime}\right)$ as [Sklar, 1959; Nelsen, 2013]

$$
p_{v}\left(v \mid v^{\prime}\right)=p_{v}(v) c\left[P_{v}(v), P_{v}\left(v^{\prime}\right)\right]
$$


where $P_{v}(v)$ is the cumulative probability

$$
P_{v}(v)=\int_{0}^{v} d v^{\prime} p_{v}\left(v^{\prime}\right)
$$

The copula $c\left(u, u^{\prime}\right)$ denotes the joint PDF of $u$ and $u^{\prime}$, whose marginals are uniform in $[0,1]$.

Inserting (31) into (26) gives

$$
\begin{aligned}
& \frac{\partial \rho(a, v ; x)}{\partial a}+v \frac{\partial \rho(a, v ; x)}{\partial x}-D \frac{\partial^{2} \rho(a, v ; x)}{\partial x^{2}} \\
& =-\frac{v}{\epsilon} \rho(a, v ; x)+\frac{v}{\epsilon} \int_{0}^{\infty} d v^{\prime} p_{v}(v) c\left[P_{v}(v), P_{v}\left(v^{\prime}\right)\right] \rho\left(a, v^{\prime} ; x\right)
\end{aligned}
$$

In the following, we employ a Gaussian copula, which is obtained from the joint Gaussian

PDF

$$
\phi\left(w, w^{\prime}\right)=\frac{\exp \left(-\frac{\mathbf{w} \mathbf{R}^{-1} \mathbf{w}}{2}\right)}{2 \pi \sqrt{\operatorname{det}(\mathbf{R})}}
$$

The correlation matrix $\mathbf{R}$ is given by

$$
\mathbf{R}=\left[\begin{array}{cc}
1 & \exp \left(-\epsilon / \ell_{c}\right) \\
\exp \left(-\epsilon / \ell_{c}\right) & 1
\end{array}\right],
$$

where $\ell_{c}$ is the correlation length along the trajectory. The joint Gaussian PDF (34) is mapped onto a joint uniform PDF according to the variable transform

$$
u=\Phi(w), \quad u^{\prime}=\Phi\left(w^{\prime}\right)
$$

where $\Phi(w)$ is the cumulative Gaussian distribution function

$$
\Phi(w)=\frac{1}{2}\left[1+\operatorname{erf}\left(\frac{w}{\sqrt{2}}\right)\right]
$$

This variable transform gives the Gaussian copula

$$
c\left(u, u^{\prime}\right)=\frac{\exp \left[-\frac{\mathbf{F}\left(u, u^{\prime}\right)\left(\mathbf{R}^{-1}-\mathbb{I}\right) \mathbf{F}\left(u, u^{\prime}\right)}{2}\right]}{\sqrt{\operatorname{det}(\mathbf{R})}}
$$

where $\mathbb{I}$ denotes the identity matrix and $\mathbf{F}\left(u, u^{\prime}\right)=\left[\Phi^{-1}(u), \Phi^{-1}\left(u^{\prime}\right)\right]^{\top}$. Figure 3.2 shows the copula function for a correlation parameter $r=\exp \left(-\epsilon / \ell_{c}\right)=10^{-1}$ (panel a), and the resulting conditional velocity distributions for log-normal marginal velocity distribution (panel b), truncated power law velocity distribution (panel c), and the conditional distribution of reciprocal velocity $\tau=1 / v$ for truncated power law marginal distribution (panel d) based on the Gaussian copula (38). 
$\mathrm{c}\left(u_{2}, u_{1}\right)$

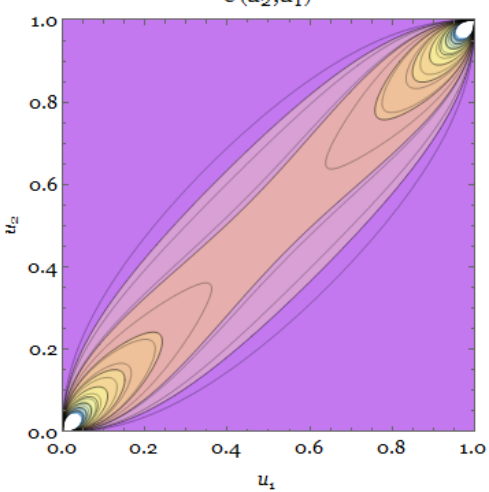

a)

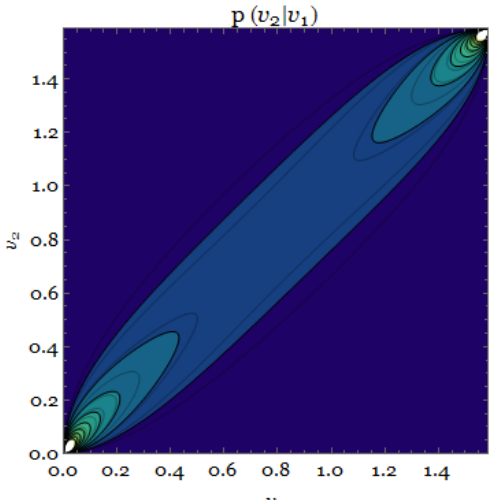

c)

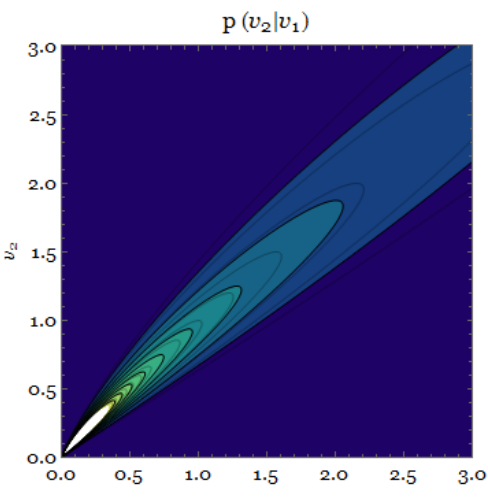

b)

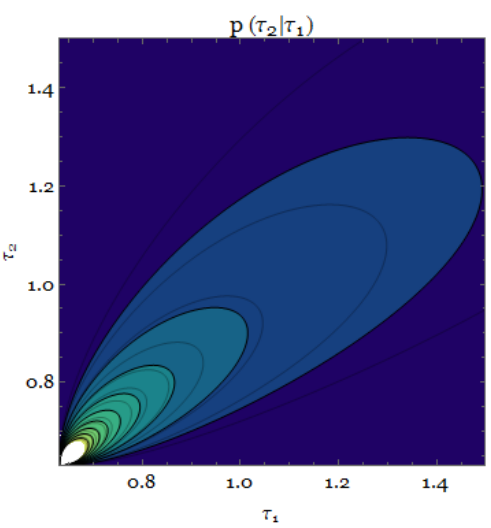

Figure 2. a) Gaussian copula with correlation factor $\mathrm{r}=0.1 \mathrm{~b}$ ) Conditional velocity distribution based on

log-normally distributed marginal velocity and Gaussian copula with correlation parameter $r \quad=\quad 0.1, \mathrm{c}$ )

Conditional velocity distribution for truncated power law marginal velocity distribution with $\alpha=5 / 2$ and

a Gaussian copula with correlation parameter of $r=0.1$ and d) Conditional reciprocal velocity distribution $\tau=1 / v$ for truncated power law marginal velocity distribution with $\alpha=5 / 2$ and Gaussian copula with correlation parameter $r=10^{-1}$. 


\subsection{Solution Methods}

In the following, we discuss solution methods for the evolution of the joint PDF of age and velocity based on the integro partial differential equation (IPDE) (33) and random walk particle tracking.

\subsubsection{IPDE Based Solution}

Instead of directly solving (33), we first perform a variable transform from velocity $v$ to the uniform random variable $u$ according to $u=P_{v}(v)$. The joint $\operatorname{PDF} \tilde{\rho}(a, u ; x)$ of $a$ and $u$ is given by

$$
\tilde{\rho}(a, u ; x)=\frac{\rho[a, v(u) ; x]}{p_{v}[v(u)]},
$$

where we defined $v(u) \equiv P_{v}^{-1}(u)$. From (33) we obtain for $\tilde{\rho}(a, u ; x)$ the evolution equation

$$
\begin{aligned}
& \frac{\partial \tilde{\rho}(a, u ; x)}{\partial a}+v(u) \frac{\partial \tilde{\rho}(a, u ; x)}{\partial x}-D \frac{\partial^{2} \tilde{\rho}(a, u ; x)}{\partial x^{2}} \\
& =-\frac{v(u)}{\epsilon} \tilde{\rho}(a, u ; x)+\frac{v(u)}{\epsilon} \int_{0}^{\infty} d u^{\prime} c\left(u, u^{\prime}\right) \tilde{\rho}\left(a, u^{\prime} ; x\right) .
\end{aligned}
$$

with the boundary condition $\tilde{\rho}(a, u ; x=0)=\delta(a)$ for $u \in[0,1]$ and 0 else. According to (41), the age distribution is obtained through marginalization, which now reads as

$$
\rho_{w}(a ; x)=\int_{0}^{1} d u \tilde{\rho}(a, u ; x) .
$$

It proves to be numerically stabler to consider the cumulative age distribution

$$
\tilde{P}(a, u ; x)=\int_{0}^{a} d a^{\prime} \tilde{\rho}\left(a^{\prime}, u, x\right) .
$$

The latter satisfies the integro partial differential equation

$$
\begin{aligned}
& \frac{\partial \tilde{P}(a, u ; x)}{\partial a}+v(u) \frac{\partial \tilde{P}(a, u ; x)}{\partial x}-D \frac{\partial^{2} \tilde{P}(a, u ; x)}{\partial x^{2}} \\
& =-\frac{v(u)}{\epsilon} \tilde{P}(a, u ; x)+\frac{v(u)}{\epsilon} \int_{0}^{\infty} d u^{\prime} c\left(u, u^{\prime}\right) \tilde{P}\left(a, u^{\prime} ; x\right)
\end{aligned}
$$

for the boundary condition $\tilde{P}(a, u ; x=0)=H(a) ; H(a)$ is the Heaviside step function. The cumulative age distribution $P_{w}(a ; x)$ is now given by

$$
P_{w}(a ; x)=\int_{0}^{1} d u \tilde{P}(a, u ; x) .
$$

Appendix C: describes a finite difference scheme for the solution of (40) and (43). 


\subsubsection{Random Walk Particle Tracking}

The solution of the transport problem in terms of random walk particle tracking is based on (12). Specifically, particle positions and age are updated according to

$$
x_{n+1}=x_{n}+\Delta s+\sqrt{2 D \Delta s} \xi_{n}, \quad a_{n+1}=a_{n}+\frac{\Delta s}{v_{n}} .
$$

with $\Delta s \ll \epsilon$. The particle velocity is updated according to (12b) as

$$
v_{n+1}=\zeta_{n} v_{n}+\left[1-\zeta_{n}\right] v\left(v_{n}\right)
$$

where $\zeta_{n}$ is 1 with probability $\exp (-\Delta s / \epsilon)$ and 0 otherwise. The conditional velocity $v(v)$ is generated by using a Gaussian copula as discussed in Section 3.2. For a given marginal PDF $p_{v}(v)$, we obtain $v(v)$ as

$$
v(v)=F^{-1}(u \mid v), \quad F(v \mid v)=\int_{0}^{P_{v}(v)} d u^{\prime} c\left[u^{\prime}, P_{v}(v)\right],
$$

where $u \in[0,1]$ is a uniformly distributed random variable. The particle age $a(x)$ is obtained as

$$
a(x)=a_{n_{x}}, \quad \quad n_{x}=\inf \left(n \mid x_{n} \leq x\right) .
$$

The age distribution $\rho_{w}(a ; x)$ is obtained from sampling $a_{n_{x}}$ as

$$
\rho_{w}(a ; x)=\left\langle\delta\left(a-a_{n_{x}}\right)\right\rangle .
$$

Figure 3.3.2 shows the normalized age distributions at two locations $x=2 \epsilon$ and $x=5 \epsilon$ for the lognormal marginal velocity distribution

$$
p_{v}(v)=\frac{\exp \left[-\frac{(\ln (v)-\mu)^{2}}{2 \sigma^{2}}\right]}{v \sqrt{2 \pi \sigma^{2}}}
$$

with a scale parameter $\sigma=1 / 2$ and a Gaussian copula with a correlation parameter of $r=$ $\exp \left(-\epsilon / \ell_{c}\right)=0.36$. It compares the age distributions obtained from particle tracking and finite difference solution of (40).

\subsection{Renormalization Scheme for Travel Times}

In order to determine travel times over large distances it is not necessary to solve for every step of the continous time random walk (45). Instead, we may take advantage of the fact that velocities are correlated over a finite distance and define coarse grained random 


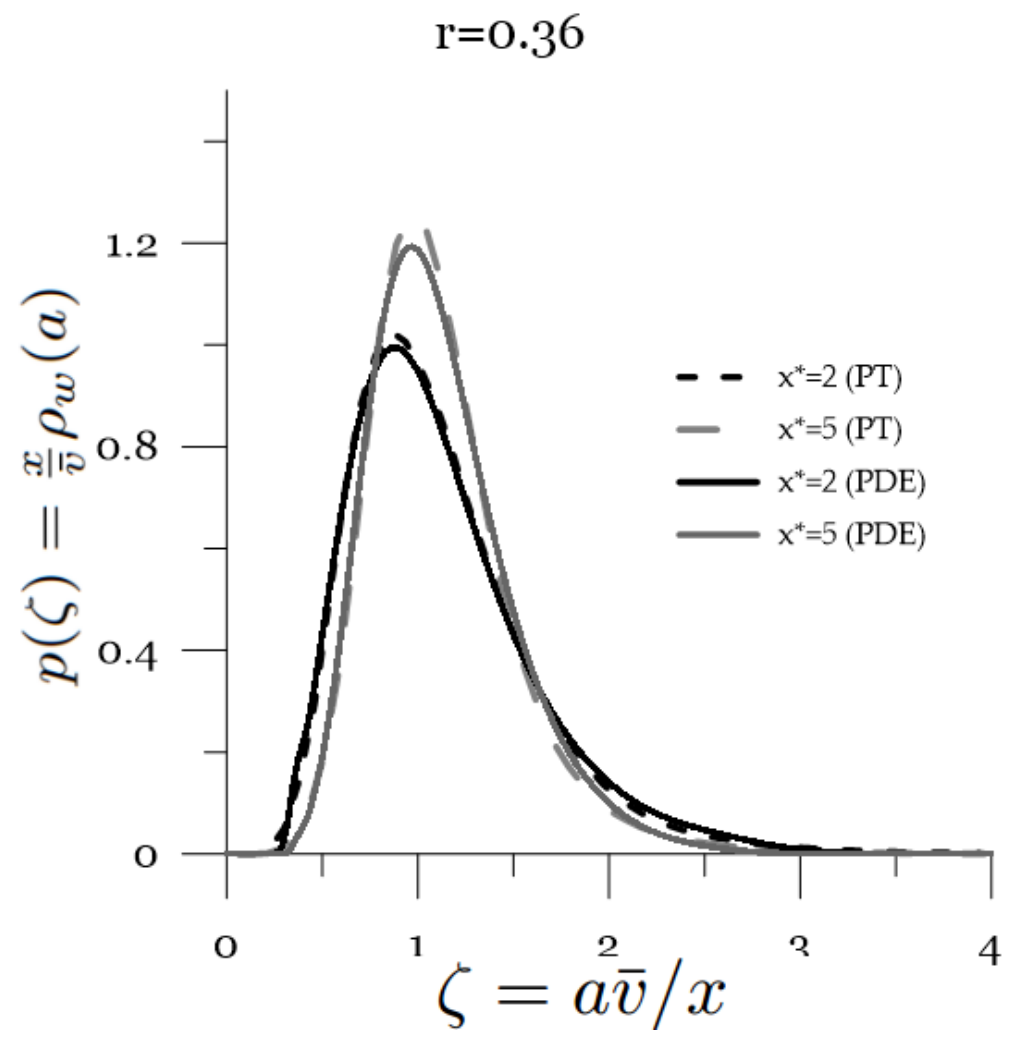

Figure 3. a) Normalized age distribution distributions at $x=2$ and $x=5$ obtained using finite difference solution of the IPDE (43) and particle tracking (PT)methods based on a log-normal marginal velocity distribution $p_{v}(v)$ and a Gaussian copula with correlation parameter $r=0.36$ 
walks that are, at large distances, characterized by the same statistics as the fine grained random walk. To this end, we first note that we have two levels of correlation. The first is due to velocity persistence on the length scale $\epsilon$, the second due to correlation on the scale $\ell_{c}$, which is encoded in the transition probability $p_{v}\left(v \mid v^{\prime}\right)$ through Gaussian copula (38) and its correlation matrix (35). We set in the following $D=0$.

\subsubsection{Coarse graining on the persistence scale $\epsilon$}

The characteristic scale for velocity changes is given by $\epsilon$. Thus, we coarse grain the random walk (45) on this scale and write

$$
x_{n+1}=x_{n}+\epsilon, \quad a_{n+1}=a_{n}+\tau_{n+1} .
$$

The transition time for a single transition with velocity $v_{n}$ is distributed according to

$$
\psi_{0}(\tau \mid v, \epsilon)=\delta(\tau-\epsilon / v)
$$

The global PDF of transition times is given by averaging of (51) over $v$ as

$$
\psi(\tau ; \epsilon)=\int_{0}^{\infty} d v \psi_{0}(\tau \mid v, \epsilon) p_{\nu}(v)=\frac{\epsilon}{\tau^{2}} p_{\nu}(\epsilon / \tau) .
$$

The conditional transition time PDF is

$$
\psi\left(\tau \mid \tau^{\prime}, \epsilon\right)=\frac{\epsilon}{\tau^{2}} p_{\nu}\left(\epsilon / \tau, \mid \epsilon / \tau^{\prime}, \epsilon\right) .
$$

where $p_{v}\left(v \mid v^{\prime}, \epsilon\right)$ is the probability to make a transition from $v_{n-1}=v^{\prime}$ to $v_{n}=v$ after a steps of length $\epsilon$. The velocity transition PDF $p_{v}\left(v \mid v^{\prime}, m \epsilon\right)$ to make a transition from $v^{\prime}$ to $v$ after $m$ steps is given in terms of the Gaussian copula (38) for the correlation matrix (see Appendix D: )

$$
\mathbf{R}_{m}=\left[\begin{array}{cc}
1 & \exp \left(-m \epsilon / \ell_{c}\right) \\
\exp \left(-m \epsilon / \ell_{c}\right) & 1
\end{array}\right],
$$

The age PDF $\rho_{w}\left(a ; x_{n}\right)$ satisfies the Kolmogorov equation

$$
\rho_{w}\left(a ; x_{n+1}\right)=\int_{0}^{a} d a^{\prime} \psi\left(a-a^{\prime} \mid a^{\prime}, \epsilon\right) \rho_{w}\left(a^{\prime}, x_{n}\right) .
$$

The age $a_{n}$ after $n$ steps is given by

$$
a_{n}=\sum_{i=1}^{n} \tau_{i}
$$


We now write this sum by defining blocks of size $m$ such that

$$
a_{n}=\sum_{k=1}^{n / m}\left(\sum_{j=1}^{m} \tau_{j, k}\right)
$$

where $n=k m$ is given in mulitples of $m$. Note that the correlation of transition times between adjacent blocks decresases with the block size $m \epsilon$. Thus, we approximate now

$$
\sum_{j=1}^{m} \tau_{j, k} \approx \hat{\tau}_{k}
$$

where the $\hat{\tau}_{k}$ are characterized by the marginal $\operatorname{PDF} \psi(\tau, m \epsilon)$ and conditional $\operatorname{PDF} \psi\left(\tau \mid \tau^{\prime}, m \epsilon\right)$ defined by (52) and (53), which account for weaker correlation of subsequent increments.

The coarse grained continous time random walk is now given by

$$
x_{k+1}=x_{k}+m \epsilon, \quad a_{k+1}=a_{k}+\hat{\tau}_{k+1} .
$$

The age distribution $\rho\left(a, x_{k}\right)$ after $k$ steps evolves according to the Kolmogorow equation

$$
\rho_{w}\left(a ; x_{k+1}\right)=\int_{0}^{a} d a^{\prime} \psi\left(a-a^{\prime} \mid a^{\prime}, m \epsilon\right) \rho_{w}\left(a^{\prime}, x_{k}\right) .
$$

We may define the block-size in a progressive way by doubling it at each step. In this case (59) becomes

$$
x_{k+1}=2 x_{k}, \quad a_{k+1}=a_{k}+\hat{\tau}_{k+1},
$$

where $x_{1}=\epsilon$. This means that $x_{k}=2^{k} \epsilon$. This CTRW is inhomogeneous in the sense that the space and time increments now depend on the step number. This is also reflected in the marginal and conditional PDFs of the transition time, which now for the $k$-th step are given by $\psi\left(\tau, 2^{k} \epsilon\right)$ and $\psi\left(\tau \mid \tau^{\prime}, 2^{k} \epsilon\right)$. The PDF of age after $k$ steps now satisfies

$$
\rho_{w}\left(a ; 2^{k+1}\right)=\int_{0}^{a} d a^{\prime} \psi\left(a-a^{\prime} \mid a^{\prime}, 2^{k} \epsilon\right) \rho_{w}\left(a^{\prime}, 2^{k}\right) .
$$

Fig. 3.4.1 shows four example prediction of the age distribution based on a lognormal velocity distribution with scale parameter of $\sigma=1 / 2$ for correlation lengths of $c_{c}=0, l_{c}=\epsilon$, $l_{c}=2 \epsilon, l_{c}=9.5 \epsilon$. We use 5 steps in the progressive renormalization (61), which corresponds to $2^{5}$ steps in the fine grained CTRW (45). The fine and coarse-grained simulation are in good agreement, which confirms the validity of the method. 

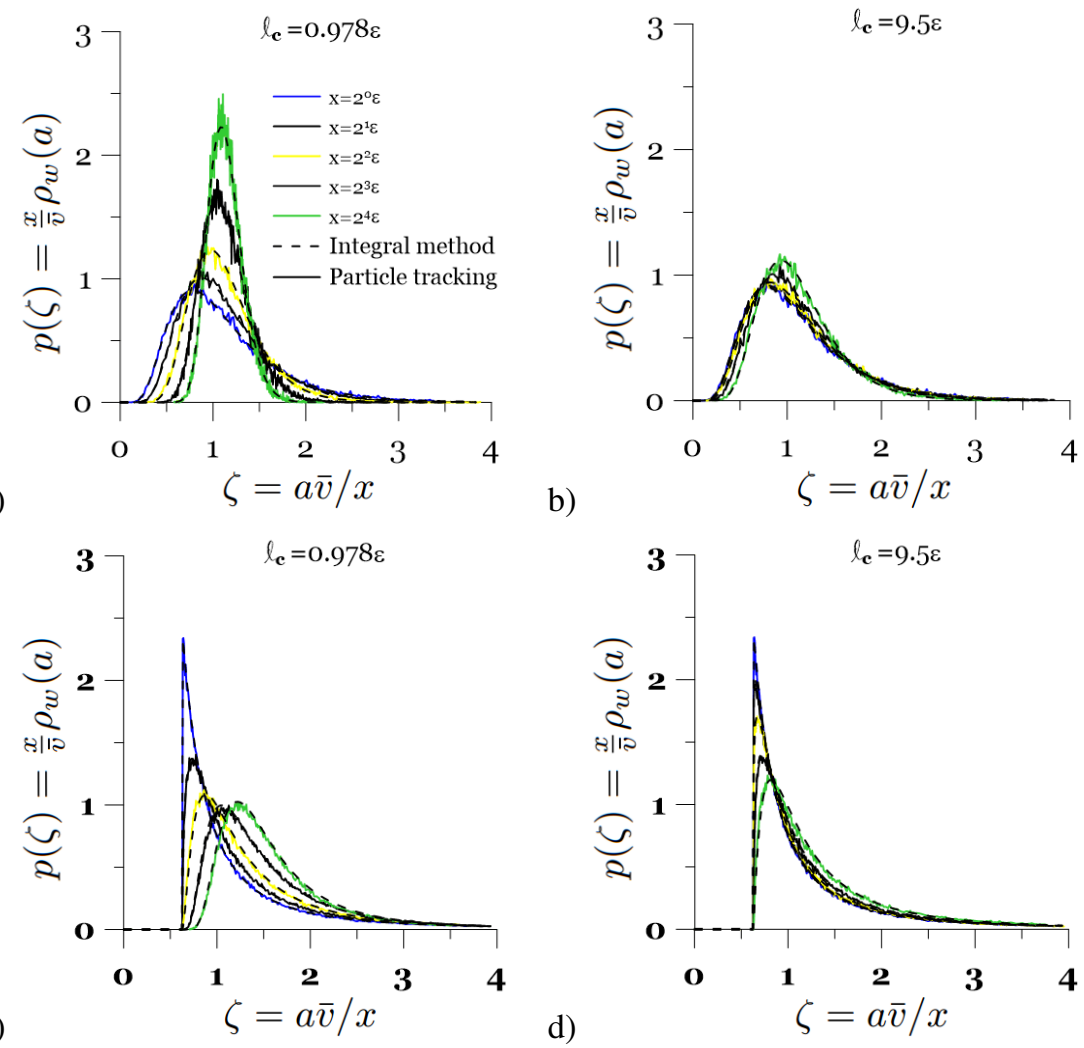

Figure 4. Normalized local age distribution obtained based on the summation technique. The solution are based on a and b) a log-normally distributed local velocity with a scale parameter of $\sigma=0.5$ and $\mathrm{c}$ and d) a truncated power law distribution with $\alpha=1.5$ and a Gaussian Copula with correlation. In panels a and $\mathrm{c} \epsilon=l_{c} / 0.978$ resulting in a correlation factor of 0.36 and in panels $\mathrm{b}$ and $\mathrm{d} \epsilon=l_{c} / 9.5$ resulting in a correlation factor of $0.9 . \bar{v}$ is the median marginal velocity. 


\subsubsection{Coarse graining on the correlation scale $\ell_{c}$}

We consider now the situation of $m \geq m_{c}=\left\lceil\ell_{c} / \epsilon\right\rceil$, where $\lceil\cdot\rceil$ denotes the ceiling function. In this case subsequent velocities can be considered independent because they are separated by distances larger than the correlation scale $\ell_{c}$. Thus, for $m>m_{c}$, we write

$$
\psi\left(\tau \mid \tau^{\prime}, m \epsilon\right)=\psi(\tau, m \epsilon)
$$

The Kolmogorow equation (55) for $\rho_{w}\left(a ; x_{k}\right)$ reduces to

$$
\rho_{w}\left(a ; x_{k+1}\right)=\int_{0}^{a} d a^{\prime} \psi\left(a-a^{\prime}, m \epsilon\right) \rho_{w}\left(a^{\prime}, x_{n}\right) .
$$

The Laplace space solution for $\rho\left(a ; x_{k}\right)$ is given by

$$
\rho_{w}^{*}\left(\lambda ; x_{k}\right)=\psi^{*}(\lambda, m \epsilon)^{k}
$$

The travel time PDF is described by an uncorrelated CTRW [Berkowitz et al., 2006]. Specficially, for $m=m_{c}$, it satisfies the generalized advection-dispersion equation [Dentz and Berkowitz, 2003; Dentz et al., 2004]

$$
\frac{\partial \rho_{w}(a ; x)}{\partial a}+\frac{\partial}{\partial a} \int_{0}^{a} d a^{\prime} \varphi\left(a-a^{\prime}\right) \rho_{w}\left(a^{\prime} ; x\right)+\left(\langle v\rangle \frac{\partial}{\partial x}-\mathcal{D} \frac{\partial^{2}}{\partial x^{2}}\right) \rho_{w}(a ; x)=0,
$$

with $\mathcal{D}=\langle v\rangle \ell_{c} / 2$. The memory function $\varphi(a)$ is given in Laplace space by

$$
\varphi(\lambda)=\frac{1-\psi_{c}^{*}(\lambda)(1-\langle\tau\rangle \lambda)}{\lambda\langle\tau\rangle \psi_{c}^{*}(\lambda)},
$$

where we set $\psi_{c}(t) \equiv \psi\left(t ; \ell_{c}\right)$. The Laplace transform is denoted by an asterisk, the Laplace variable is $\lambda$.

\section{Impact of velocity correlation and distribution on the distribution of groundwa- ter age}

In this section, the impact of correlation between subsequent velocities, and their marginal distribution on the distribution of age is studied. To this end, we consider three different marginal velocity distributions, which give rise to different long-time behaviors of the age distributions. We employ the the lognormal distribution (49). Furthermore we use the truncated power-law velocity PDF

$$
p_{v}(v)=\frac{\alpha}{v_{c}}\left(\frac{v}{v_{c}}\right)^{\alpha-1} H\left(v_{c}-v\right)
$$

where $v_{c}$ is an upper cutoff, $\alpha>0$, and $H(v)$ is the Heaviside step function. We also consider the inverse Lévy distribution

$$
p_{v}(v)=\frac{\exp \left(-\frac{v}{2 v_{c}}\right)}{\sqrt{2 \pi v_{c} v^{1 / 2}}}
$$


The correlated velocity series are generated as described in Sections 3.2 using a Gaussian copula. Correlation is measured in terms of the correlation length $\ell_{c}$ of the resulting Markov chain. We employ the numerical particle tracking approach described in Section 3.3.2 using $10^{6}$ particles, $\epsilon=1$ and $\Delta s=2.5 \times 10^{-2}$. Furthermore, we set $D=0$.

Figure 4 illustrates the distribution of travel times for lognormal and power-law velocity PDFs at different distances from the inlet boundary. At distances $x$ from the boundary shorter or of the order of the correlation length $\ell_{c}$, velocities as strongly correlated. The system behaves ballistically (particle velocities are essentially constant), and is equivalent to a stochastic streamtube approach, as discussed in Section 3 and in Kang et al. [2015]. Thus, the age distribution for $x \leq \ell_{c}$ can be approximated by (30), this means they are fully determined in terms of the velocity PDF and equal to the travel time PDF (52) for $\epsilon=x$. These behaviors can be clearly seen in Figures $4-4$ for distances $x / \ell_{c} \leq 1$.

In the opposite limit of $x \gg \ell_{c}$, velocities decorrelate as discussed in Section (3.4). The travel times then describe an uncorrelated CTRW. The evolution of the age PDF is given by (64). If we choose $\ell_{c}$ as the coarse-graining scale as discussed in Section 3.4, the age CTRW is fully characterized by the transition time PDF (52) for $\epsilon=\ell_{c}$. For the lognormal velocity $\mathrm{PDF}$, the corresponding transition time $\operatorname{PDF} \psi\left(t ; \ell_{c}\right)$ is also lognormal. The truncated power-law velocity PDF (68) gives a transition time PDF that behaves as

$$
\psi\left(t ; \ell_{c}\right) \propto t^{-1-\alpha}
$$

which for $\alpha=1 / 2$ gives the long time behavior corresponding to the Lévy PDF (69). For the lognormal PDF and the power-law PDF with $\beta>2$, the age distribution converges towards an inverse Gaussian according to the central limit theorem. This can be observed in Figures 4. Note specficially, that the power-law velocity PDF with $\alpha>2$ gives a power-law age PDF at short distances, but at larger distances converges to an inverse Gaussian PDF. For $0<\alpha<2$ in the power-law transition time PDF (70), the generalized central limit theorem gives a stable age PDF at large distances,

$$
\rho_{w}(a, x) \propto a^{-1-\alpha}
$$

This behavior is illustrated in Figure 4 for $x \gg \ell_{c}$. At intermediate distances, the age distributions evolve from the streamtube limit to the respective stable limit distributions when $x \gg \ell_{c}$. 
$l_{c}=\varepsilon, \alpha=2.5$

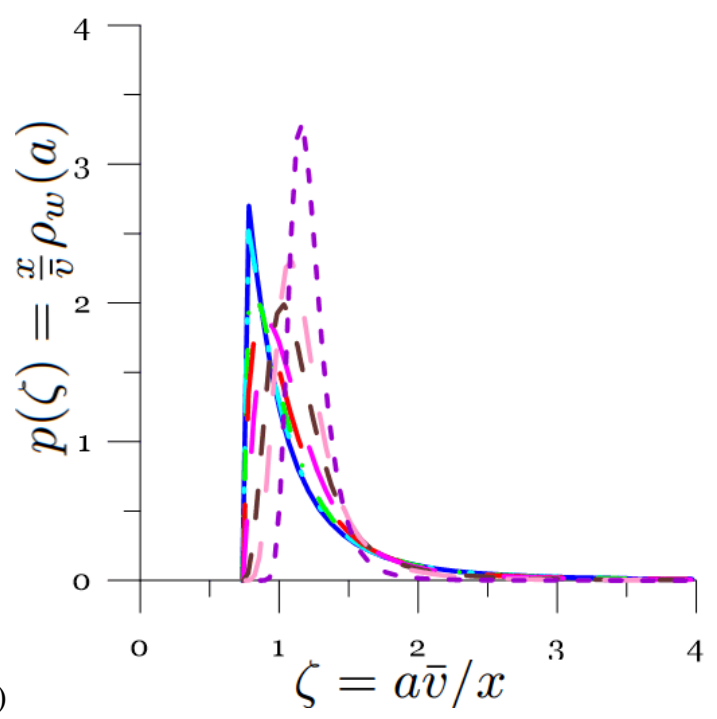

a)

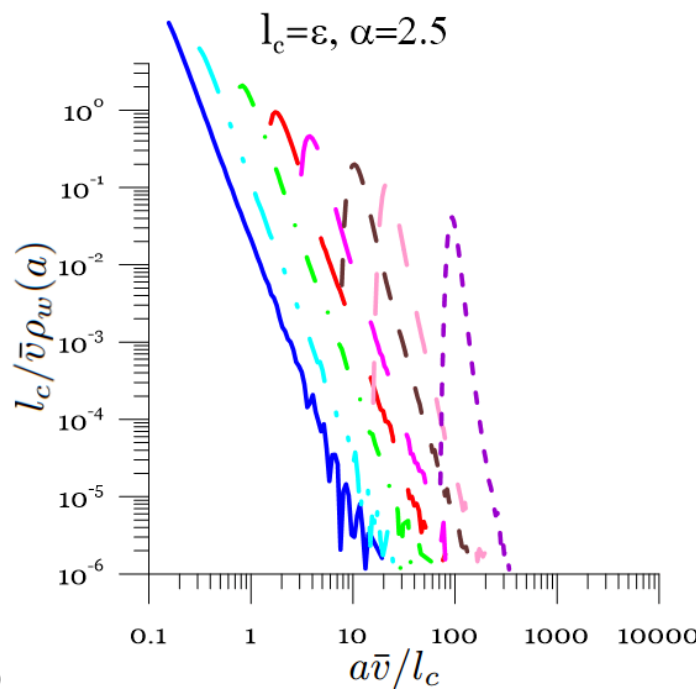

$l_{c}=\varepsilon$, Lognormal

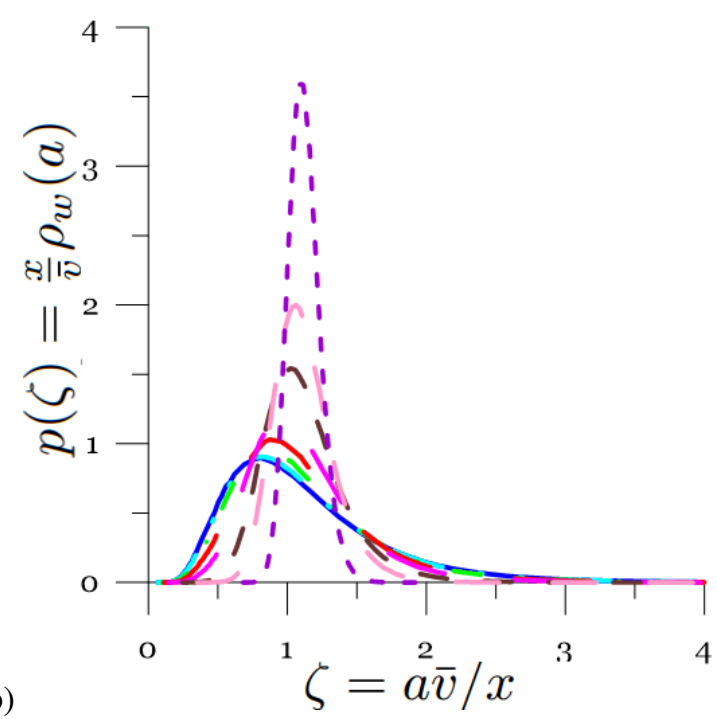

b)

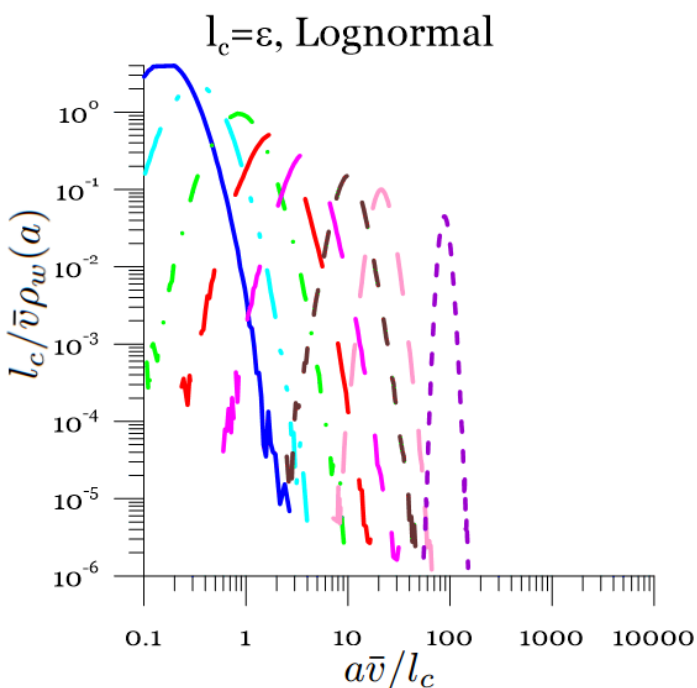

d)

$$
\begin{aligned}
&-x / l_{c}=0.2- x / l_{c}=1-x / l_{c}=4-x / l_{c}=20 \\
&-x / l_{c}=0.4-x / l_{c}=2--x / l_{c}=10-x / l_{c}=80
\end{aligned}
$$

Figure 5. Normalized travel time distribution obtained using particle tracking based on Gaussian copula with an $\epsilon=\ell_{c}$ and a and c) truncated power-law distribution with $\alpha=2.5$ and b and d) Lognormal distribution with a shape factor $\sigma=0.5 \cdot 10^{6}$ particles were used to produce the results. $\bar{v}$ is the median marginal velocity. 

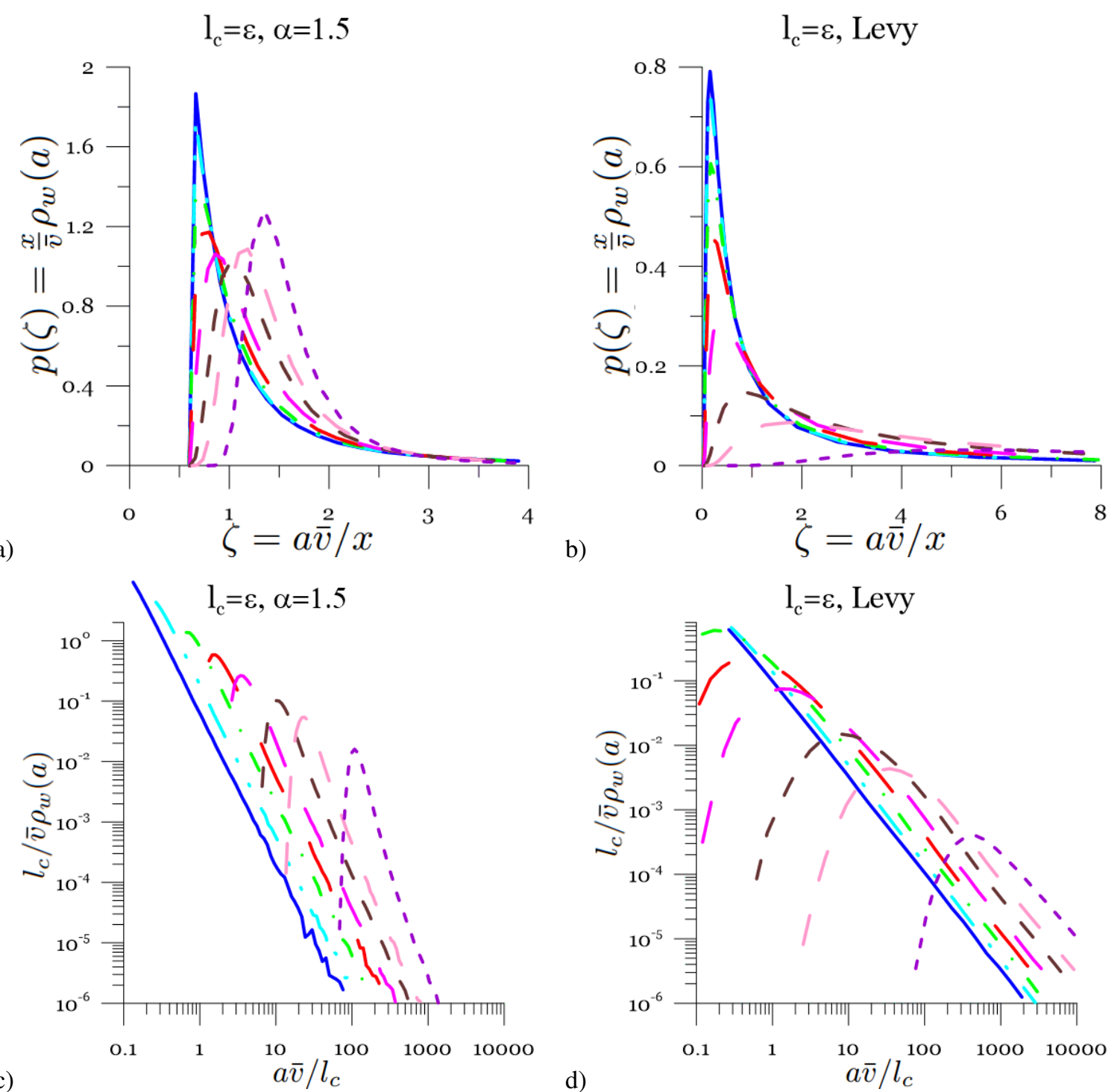

d)

$$
\begin{aligned}
&-x / l_{c}=0.2-\cdot x / l_{c}=1-x / l_{c}=4-x / l_{c}=20 \\
&-x / l_{c}=0.4-x / l_{c}=\mathbf{2}-x / l_{c}=10-x / l_{c}=80
\end{aligned}
$$

Figure 6. Normalized travel time distribution obtained using particle tracking based on Gaussian copula with $\epsilon=\ell_{c}$ and a and c) truncated power-law distribution with $\alpha=1.5$ and $\mathrm{b}$ and d) Levy distribution. $10^{6}$ particles were used to produce the results. $\bar{v}$ is the median marginal velocity. 

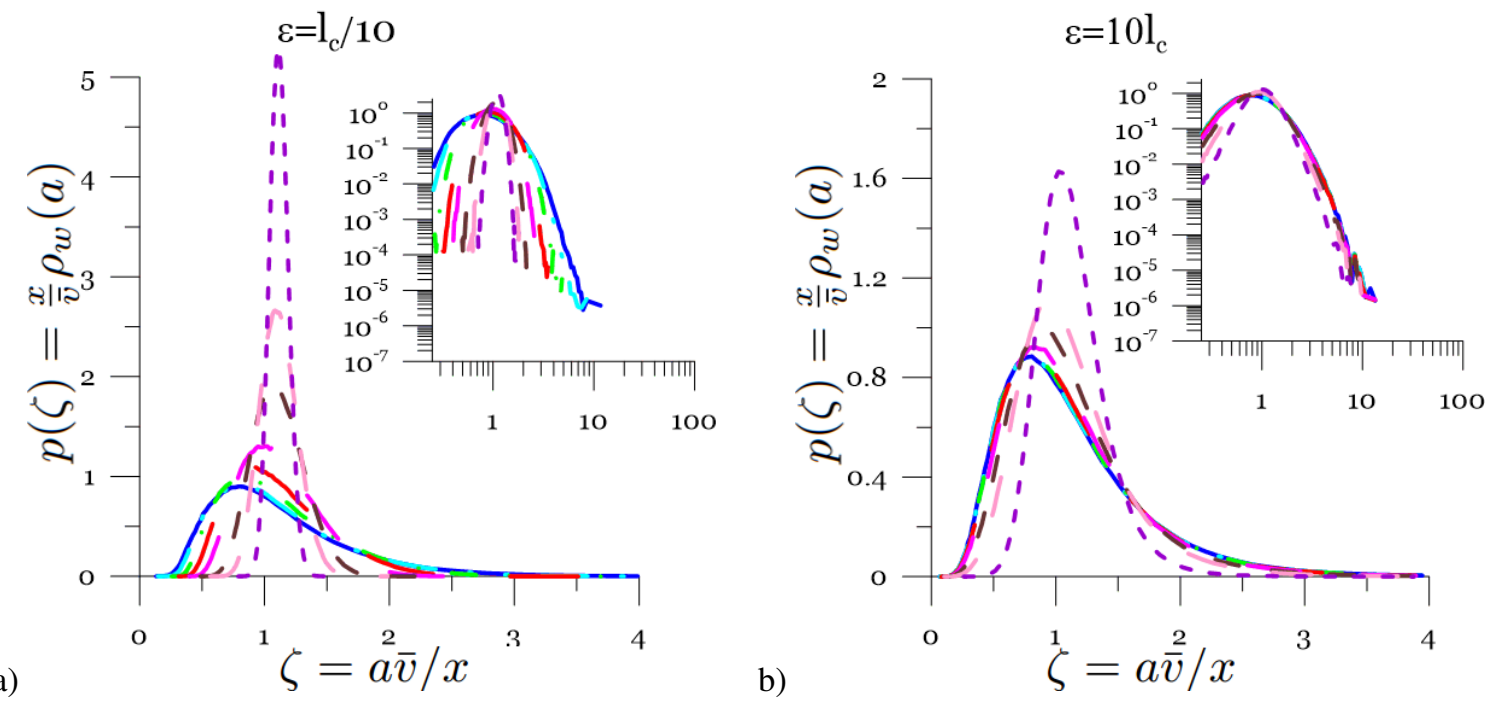

a)

$$
\begin{aligned}
&-x / l_{c}=0.2- x / l_{c}=1-x / l_{c}=4-x / l_{c}=20 \\
&-x / l_{c}=0.4-x / l_{c}=2--x / l_{c}=10-x / l_{c}=80
\end{aligned}
$$

Figure 7. Sensitivity with respect to $\epsilon$. The figure shows normalized travel time distribution obtained using particle tracking based on Gaussian copula with a) $\epsilon=\ell_{c} / 10$ and b) $\epsilon=10 \ell_{c}$ for a log-normally distributed marginal velocity distribution with $\sigma=0.5 \cdot 10^{6}$ particles were used to produce the results. $\bar{v}$ is the median marginal velocity

Figure 4 illustrates the impact of the persistence $\epsilon$ on the age distribution. The intrinsic length $\epsilon$ sets the characteristic scale at which particle velocities change. At distances smaller than $\epsilon$ velocities are approximately constant. This velocity variability on the scale $\epsilon$ gives rise to an intrinsic dispersion effect $\propto \epsilon$ [Dentz et al., 2016]. For $\epsilon \gg \ell_{c}$ the correlation scale plays no role and the evolution of the age distribution is fully determined by $\epsilon$.

\section{Summary and Conclusions}

We studied the evolution of the joint distribution of age and velocity of fluid particles based on a Markov model for fluid velocities as a function of travel distance along streamlines [Dentz et al., 2016]. The transition probability between subsequent particle velocities is determined based on the known local velocity distribution and velocity correlation as specified by a copula function. The evolution of fluid particles in this framework described a CTRW. The joint PDF of age, arrival time and particle velocity is governed by a Boltzmanntype integro-partial differential equation.This approach quantifies both the impact of velocity correlation and velocity distribution on the evolution of the distirbution of groundwater age. 
We study the distribution of arrival times at distances larger than the intrincis correlation length scales using a renomalization scheme for particle transition times. For distances much larger than the velocity correlation length, we show that the travel time distribution can be described by an uncorrelated CTRW.

For distances close to the inlet, the approach is equivalent to the stochastic streamtube approach because particle velocities are strongly correlated along streamlines. In this case, the age distribution is obtained by a direct map from the velocity distribution. At increasing distance relative to the correlation length the age distribution evolves according to the (generalized) central limit theorem. For velocity distributions that correponds to transition times with finite variance, the age distribution evolves towards an inverse Gaussian. For velocity distribution that are skewed towards low velocities and thus gives rise to heavy tailed transition time distribution, the age distribution adjusts according to the generalized central limit theorem and evolves in space towards a stable distribution.

The proposed framework can be also applied to contaminant transport problems in correlated velocity fields [Dentz et al., 2016]. It can be potentially used for extracting velocity correlation and local velocity distributions by utilizing tracer studies or multiple spatially distributed age tracers via inverse modeling.

\section{A: Derivation of the Governing Equation for the Joint PDF of Arrival Time, Age and Velocity}

We detail here the derivation of the governing equation (19) for the joint density $p\left(t, a, v ; x \mid v_{0}\right)$. To this end, we first note that the first particle arrival at the position $x$ is defined by

$$
t(x) \equiv t[\sigma(x)], \quad \sigma(x)=\inf \{s \mid x(s) \geq x\},
$$

where $\sigma(x)$ is the $s$ of first arrival for $x(s)$ at $x$ according to (12a). Accordingly, we obtain for age and particle velocity,

$$
a(x) \equiv a_{0}+t[\sigma(x)]-t_{0}, \quad v(x)=v[\sigma(x)],
$$

where the time of injection at $x=0$ is $t(x=0)=t_{0}$, the particle age at the boundary is $a(x=$ $0)=a_{0}$, which we set to 0 here. Age $a$ here is equal to the travel time from the boundary to the position $x$. Note that $\sigma(x)$ describes a first-passage problem in a constant velocity field with the $s$-dependent diffusion coefficient $D / v(s)$. Thus, the distribution $f(s, x)=\langle\delta[s-$ $\sigma(x)]\rangle$, where the angular brackets here denote the noise-averaging over $\xi(s)$, satisfies the 
advection-diffusion equation [Kreft and Zuber, 1978]

$$
\frac{\partial f(s, x)}{\partial s}=-\frac{\partial f(s, x)}{\partial x}+\frac{D}{v(s)} \frac{\partial^{2} f(s, x)}{\partial x^{2}} .
$$

The joint distribution of time, age and velocity at a position $x$ is given by

$$
\rho(t, a, v ; x)=\langle\delta[t-t[\sigma(x)]] \delta[a-a(t)] \delta[v-v[\sigma(x)]]\rangle
$$

As above, due to homogeneity in time, the joint PDF depends only on the difference $t-t_{0}$.

Thus, for simplicity, we set $t_{0}=0$. Expanding (A.4), the joint density can be written as

$$
\rho(t, a, v ; x)=\int_{0}^{\infty} d s R(t, a, v, s ; x),
$$

where the $R(t, a, v, s ; x)$ is the joint PDF of $(a, t, v, s)$ at a location $x$ which is defined as,

$$
R(t, a, v, s ; x)=\langle\delta[a-a(s)] \delta[t-t(s)] \delta[v-v(s)] f(s, x)\rangle
$$

As the processes $t(s), v(s)$ and $\sigma(x)$ are Markovian, $R(t, a, v, s ; x)$ satisfies the Kolmogorov equation

$$
\begin{aligned}
& R(t, a, v ; s+\Delta s, x)= \\
& \int_{0}^{\infty} d v^{\prime} r\left(v \mid v^{\prime}\right)\left\langle\delta\left[a-a(s)-\Delta s / v^{\prime}\right] \delta\left[t-t(s)-\Delta s / v^{\prime}\right] \delta[v-v(s)] f(x, s+\Delta s)\right\rangle
\end{aligned}
$$

We set $f(x, s+\Delta s)=f(x, s)+\Delta s \partial f(x, s) / \partial s+\ldots$, and use (A.3) for the $s$-derivative of $f(x, s)$. This gives

$$
\begin{aligned}
& R(t, a, v ; s+\Delta s, x)= \\
& \int d v^{\prime} r\left(v \mid v^{\prime}\right)\left(1-\Delta s \frac{\partial}{\partial x}+\Delta s \frac{D}{v^{\prime}} \frac{\partial^{2}}{\partial x^{2}}\right) R\left(t-\Delta s / v^{\prime}, a-\Delta s / v^{\prime}, v^{\prime} ; s, x\right)+\ldots,
\end{aligned}
$$

where the dots denote contributions of order $\Delta s^{2}$. We expand $R\left(t-\Delta s / v^{\prime}, a-\Delta s / v^{\prime}, v^{\prime} ; s, x\right)$ up to linear oder in $\Delta s$, which gives

$$
\begin{aligned}
& R(t, a, v ; s+\Delta s, x)=\int d v^{\prime} r\left(v \mid v^{\prime}\right)\left(1-\Delta s \frac{\partial}{\partial x}+\Delta s \frac{D}{v^{\prime}} \frac{\partial^{2}}{\partial x^{2}}\right) R\left(t, a, v^{\prime} ; s, x\right) \\
& -\int d v^{\prime} r\left(v \mid v^{\prime}\right) \frac{\Delta s}{v^{\prime}}\left(\frac{\partial}{\partial a}+\frac{\partial}{\partial t}\right) R\left(t, a, v^{\prime} ; s, x\right)+\ldots
\end{aligned}
$$

where the dots indicate terms of order $\Delta s^{2}$. Inserting the explicit form (15) for $r\left(v \mid v^{\prime}\right)$ gives

$$
\begin{aligned}
& R(t, a, v ; s+\Delta s, x)=\exp (-\Delta s / \epsilon)\left(1-\Delta s \frac{\partial}{\partial x}+\Delta s \frac{D}{v} \frac{\partial^{2}}{\partial x^{2}}\right) R(t, a, v ; s, x) \\
& -\exp (-\Delta s / \epsilon) \frac{\Delta s}{v}\left(\frac{\partial}{\partial a}+\frac{\partial}{\partial t}\right) R(t, a, v ; s, x) \\
& +[1-\exp (-\Delta s / \epsilon)] \int d v^{\prime} p_{v}\left(v \mid v^{\prime}\right) R\left(t, a, v^{\prime} ; s, x\right)+\ldots
\end{aligned}
$$


Expanding the remaining terms consistently up to order $\Delta s$ gives

$$
\begin{aligned}
& R(t, a, v ; s+\Delta s, x)=R(t, a, v ; s, x)-\frac{\Delta s}{\epsilon} R(t, a, v ; s, x)-\Delta s\left(\frac{\partial}{\partial x}-\frac{D}{v} \frac{\partial^{2}}{\partial x^{2}}\right) R(t, a, v ; s, x) \\
& +\frac{\Delta s}{v}\left(\frac{\partial}{\partial a}+\frac{\partial}{\partial t}\right) R(t, a, v ; s, x)+\frac{\Delta s}{\epsilon} \int d v^{\prime} p_{v}\left(v \mid v^{\prime}\right) R\left(t, a, v^{\prime} ; s, x\right)+\ldots
\end{aligned}
$$

In the limit of $\Delta s \rightarrow 0$, we obtain

$$
\begin{aligned}
& \frac{\partial R(t, a, v ; s, x)}{\partial s}=-\frac{1}{\epsilon} R(t, a, v ; s, x)-\left(\frac{\partial}{\partial x}-\frac{D}{v} \frac{\partial^{2}}{\partial x^{2}}\right) R(t, a, v ; s, x) \\
& -\frac{1}{v}\left(\frac{\partial}{\partial a}+\frac{\partial}{\partial t}\right) R(t, a, v ; s, x)+\frac{1}{\epsilon} \int d v^{\prime} p_{v}\left(v \mid v^{\prime}\right) R\left(t, a, v^{\prime} ; s, x\right)
\end{aligned}
$$

Integration of this equation over $s$ according to (A.5) gives the governing equation (19).

\section{B: Derivation of the governing equation for the evolution of the joint distribution local age, velocity and position using Reynold's transport theorem}

Above and in the remainder of the paper, we consider the joint distribution of age and velocity at a position $x$ and at time $t$, denoted by $\rho_{a}(a, v ; x, t)$. Here we want to briefly consider the derivation of a governing equation of the joint distribution of age, velocity and position at a given time $t$ denoted in the following by $\rho_{s}(a, v, x ; t)$. Thus, let us assume transport of water molecules in a three-dimensional framework with coordinate $(x, a, v)^{\top}$ in which the $a-$ and $v$-axes are orthogonal to the $x$-axis. The amount of water in a differential block with dimensions $\Delta x \times \Delta v \times \Delta a$ is $\approx \rho_{s}(a, v, x ; t) \Delta x \Delta v \Delta a$. This is mass of water with an age within $[a-\Delta a / 2, a+\Delta a / 2]$ a velocity within $[v-\Delta v / 2, v+\Delta v / 2]$ and a location within $[x-\Delta x / 2, x+\Delta x / 2]$. So we can write the mass balance for this control volume considering influxes and outfluxes including, aging, advection and Fickian diffusion and exchange between flow paths with different velocities (i.e., velocity bins). Let us further assume that the rate coefficient of leaving a velocity bin is $k(v)[1 / \mathrm{T}]$ and the probability of moving from a velocity bin to another one is determined by the conditional PDF $p\left(v \mid v^{\prime}\right)$. The water balance equation for

$$
\begin{aligned}
& \frac{\Delta \rho_{s}(a, v, x ; t)}{\Delta t} \Delta x \Delta v \Delta a=\rho_{s}(a-\Delta a, v, x ; t) \Delta v \Delta x-\rho_{s}(a, v, x ; t) \Delta v \Delta x \\
& +D \frac{\rho_{s}(a, v ; x-\Delta x ; t)-\rho_{s}(a, v, x ; t)}{\Delta x} \Delta a \Delta v+D \frac{\rho_{s}(a, v, x+\Delta x ; t)-\rho_{s}(a, v, x ; t)}{\Delta x} \Delta a \Delta v \\
& +\left[v \rho_{s}(a, v, x-\Delta x ; t)-v \rho_{s}(a, v, x ; t)\right] \Delta a \Delta v \\
& +\sum_{i} k\left(v_{i}\right) p\left(v \mid v_{i}\right) \rho_{s}\left(a, v_{i} ; x, t\right) \Delta v \Delta a \Delta x-k(v) \rho_{s}(a, v, x ; t) \Delta v \Delta a \Delta x
\end{aligned}
$$


Dividing (B.1) by $\Delta x \Delta v \Delta a$ and taking the limit $\Delta x \rightarrow 0$ and $\Delta v \rightarrow 0, \Delta a \rightarrow 0$ gives

$$
\begin{aligned}
\frac{\partial}{\partial t} \rho_{s}(a, v, x ; t) & =-\frac{\partial}{\partial a} \rho_{s}(a, v, x ; t)-v \frac{\partial}{\partial x} \rho_{s}(a, v, x ; t) \\
& +D \frac{\partial^{2} \rho_{s}(a, v, x ; t)}{\partial x^{2}}+\int d v^{\prime} k\left(v^{\prime}\right) p\left(v \mid v^{\prime}\right) \rho_{s}\left(a, v^{\prime}, x ; t\right)-k(v) \rho_{s}(a, v, x ; t)
\end{aligned}
$$

Now defining $\rho_{s}(a, v ; x, t)$ as [Pope, 2001]:

$$
\rho_{s}(a, v ; x, t)=\rho_{s}(a, v \mid x, t)=\frac{\rho_{s}(a, v, x ; t)}{\rho_{s}(x ; t)}
$$

and considering the fact that $\rho_{s}(x ; t)=\iint d v d a \rho_{s}(a, v, x ; t)$ represents the density of water that under an incompressibility assumption is constant, Eq. (B.2) can be written as:

$$
\begin{aligned}
\frac{\partial}{\partial t} \rho_{s}(a, v ; x, t) & =-\frac{\partial}{\partial a} \rho_{s}(a, v ; x, t)-v \frac{\partial}{\partial x} \rho_{s}(a, v ; x, t) \\
& +D \frac{\partial^{2} \rho_{s}(a, v, x ; t)}{\partial x^{2}}+\int d v^{\prime} k\left(v^{\prime}\right) p\left(v \mid v^{\prime}\right) \rho_{s}\left(a, v^{\prime} ; x, t\right)-k(v) \rho_{s}(a, v ; x, t)
\end{aligned}
$$

By setting $k(v)=v / \epsilon$, we obtain the transport equation corresponding to (23). Note however the difference in the integral terms governing the velocity transitions. Here, the frequency of velocity changes depends on the previous particle velocity, while in (23) it depends on the current particle velocity. This is due to the fact that (23) describes the evolution of the joint distribution of age and velocity in space. In fact, we may write (23) as

$$
\begin{aligned}
\frac{\partial \rho_{a}(a, v ; x, t)}{\partial x} & =-\frac{1}{v}\left(\frac{\partial}{\partial a}+\frac{\partial}{\partial t}\right) \rho_{a}(a, v ; x, t) \\
& -\epsilon^{-1} \rho_{a}(a, v ; x, t)+\epsilon^{-1} \int d v^{\prime} p_{v}\left(v \mid v^{\prime}\right) \rho_{a}\left(a, v^{\prime} ; x, t\right),
\end{aligned}
$$

where we disregard diffusion for illustration. This form of (23) emphasizes the fact that it describes an evolution in space. Velocity changes thus occur by a constant spatial rate, which here is $1 / \epsilon$. Equation (B.2) on the other hand considers the change of the distribution of age and vleocity in a control volume.

\section{C: A numerical algorithm for the solution of the correlated advection-dispersion equation}

Eqs. (40) and (43) can be solved using finite difference approximation by discretizing the $u, a$, and $x$ coordinates into nodes:

$$
\tilde{\rho}\left(a_{i}, u_{j} ; x_{k}\right) \equiv \tilde{\rho}_{i, j, k}, \quad \quad v\left(u_{j}\right) \equiv v_{j}, \quad c\left(u, u^{\prime}\right)=c_{j, j^{\prime}},
$$

and for $\tilde{P}(a, u ; x)$ accordingly. Using Euler's backward approximation in $a$ and upwind descretization with Crank-Nicholson marching in $x$, (40) and (43) can be written in discretized form as 


$$
\begin{aligned}
& \frac{\tilde{\rho}_{i, j, k}-\tilde{\rho}_{i-1, j, k}}{\Delta a}+v_{j} \frac{\tilde{\rho}_{i-1 / 2, j, k}-\tilde{\rho}_{i-1 / 2, j, k-1}}{\Delta x} \\
& =D \frac{\tilde{\rho}_{i-1 / 2, j, k-1}-2 \tilde{\rho}_{i-1 / 2, j, k}+\tilde{\rho}_{i-1 / 2, j, k+1}}{\Delta x^{2}} \\
& +\frac{v_{j}}{\epsilon} \sum_{j^{\prime}} \Delta u c_{j, j^{\prime}} \tilde{\rho}_{i-1 / 2, j^{\prime}, k}-\frac{v_{j}}{\epsilon} \tilde{\rho}_{i-1 / 2, j, k}
\end{aligned}
$$

Equation (C.2) can be written in matrix form as

$$
\begin{aligned}
& \frac{\tilde{\boldsymbol{\rho}}_{i, k}-\tilde{\boldsymbol{\rho}}_{i-1, k}}{\Delta a}+\mathbf{v}^{\top} \frac{\tilde{\boldsymbol{\rho}}_{i-1 / 2, k}-\tilde{\boldsymbol{\rho}}_{i-1 / 2, k}}{\Delta x} \\
& =D \frac{\tilde{\boldsymbol{\rho}}_{i-1 / 2, k-1}-2 \tilde{\boldsymbol{\rho}}_{i-1 / 2, k}+\tilde{\boldsymbol{\rho}}_{i-1 / 2, k+1}}{\Delta x^{2}} \mathbf{K} \tilde{\boldsymbol{\rho}}_{i-1 / 2, k}
\end{aligned}
$$

where we defined

$$
\tilde{\boldsymbol{\rho}}_{i, k}=\left(\rho_{i, 1, k}, \ldots, \rho_{i, j, k}, \ldots\right)^{\top}, \quad \quad \mathbf{v}=\left(v_{1}, \ldots, v_{j}, \ldots\right)^{\top},
$$

and the product

$$
\mathbf{v}^{\top} \tilde{\boldsymbol{\rho}}_{i, k}=\left(v_{1} \rho_{i, 1, k}, \ldots, v_{j} \rho_{i, j, k}, \ldots\right)^{\top} .
$$

We furthermore defined the matrix

$$
\mathbf{K}=\frac{\Delta u}{\epsilon} \mathbf{v}^{\top} \mathbf{c}-\frac{\mathbf{v}^{\top}}{\epsilon}
$$

$\tilde{\boldsymbol{\rho}}_{j-1 / 2, k}$ is approximated using the Crank-Nicholson method as

$$
\tilde{\boldsymbol{\rho}}_{i-1 / 2, k}=a \tilde{\boldsymbol{\rho}}_{i-1, k}+(1-a) \tilde{\boldsymbol{\rho}}_{i, k},
$$

where $0<a<1$. This turns (C.3) into a system of linear equations that can be solved by time-marching over $a$. The fact that the stiffness matrix is independent of location $x$, allows computing the inverse of the system matrix once and then using it throughout the solution by simple multiplication. The age distribution at a given location is calculated as

$$
\rho_{w}\left(a_{i} ; x_{k}\right)=\sum_{j} \Delta u \tilde{\rho}_{i, j, k}
$$

\section{D: Evolution of Gaussian Copula-Induced Velocity Correlation}

The particle velocity $v_{n}$ is modeled as a Markov process, whose transition probability $p_{v}\left(v \mid v^{\prime}\right)$ of subsequent velocities is given by the Gaussian copula (38) with the correlation matrix (35). The transition probability $p_{v}\left(v, 2 \epsilon \mid v^{\prime}\right)$ separated by the distance $2 \epsilon$ can be obtained from the Kolmogorov equation as

$$
p_{\nu}\left(v, 2 \epsilon \mid v^{\prime}\right)=\int_{0}^{\infty} d y p_{\nu}(v \mid y) p_{v}\left(y \mid v^{\prime}\right) .
$$


Using the representation (31) in terms of the velocity copula, the latter can be written as

$$
p_{v}(v) c_{2}\left[P_{v}(v), P_{v}\left(v^{\prime}\right)\right]=\int_{0}^{\infty} d y p_{v}(v) c\left[P_{v}(v), P_{v}(y)\right] p_{v}(y) c\left[P_{v}(y), P_{v}\left(v^{\prime}\right)\right] .
$$

where $c_{2}\left(u, u^{\prime}\right)$ is the copula for the joint PDF of $v$ and $v^{\prime}$. Transformation of variables according to $z=P_{v}(y)$ gives

$$
c_{2}\left(u, u^{\prime}\right)=\int_{0}^{1} d z c(u, z) c\left(z, u^{\prime}\right)
$$

Performing the variable transform $z=\Phi(w)$, where $\Phi(w)$ is given by (37) and using the Gaussian copula (38) gives

$$
\begin{aligned}
c_{2}\left(u, u^{\prime}\right) & =\int_{-\infty}^{\infty} d w \frac{\exp \left\{-\frac{\left[\Phi^{-1}(u), w\right]^{\top}\left(\mathbf{R}^{-1}-\mathbb{I}\right)\left[\Phi^{-1}(u), w\right]^{\top}}{2}\right\}}{\sqrt{\operatorname{det}(\mathbf{R})}} \\
& \times \frac{\exp \left\{-\frac{\left[\Phi^{-1}(u), w\right]^{\top} \mathbf{R}^{-1}\left[\Phi^{-1}(u), w\right]^{\top}-\Phi^{-1}(u)^{2}}{2}\right\}}{\sqrt{2 \pi \operatorname{det}(\mathbf{R})}}
\end{aligned}
$$

Evaluating the Gaussian integral in $w$ gives

$$
c_{2}\left(u, u^{\prime}\right)=c\left(u, u^{\prime} ; 2 \epsilon\right)
$$

where $c\left(u, u^{\prime} ; 2 \epsilon\right)$ is the Gaussian copula (38) characterized by the correlation matrix (54) for $m=2$. It follows that

$$
p_{v}(v, 2 \epsilon)=p_{v}(v) c\left(u, u^{\prime} ; 2 \epsilon\right)
$$

By induction, we obtain that

$$
p_{v}\left(v, m \epsilon \mid v^{\prime}\right)=p_{v}(v) c\left[P_{v}(v), P_{v}\left(v^{\prime}\right) ; m \epsilon\right]
$$

\section{Acknowledgments}

MD acknowledges the support of the European Research Council (ERC) through the project MHetScale (617511).

\section{References}

Alikhani, J., A. L. Deinhart, A. Visser, R. K. Bibby, R. Purtschert, J. E. Moran,

A. Massoudieh, and B. K. Esser, Nitrate vulnerability projections from bayesian inference of multiple groundwater age tracers, Journal of Hydrology, doi: http://dx.doi.org/10.1016/j.jhydrol.2016.04.028, 2016. 
Bárdossy, A., and J. Li, Geostatistical interpolation using copulas, Water Resources Research, 44(7), 2008.

Berkowitz, B., A. Cortis, M. Dentz, and H. Scher, Modeling non-fickian transport in geological formations as a continuous time random walk, Rev. Geophys., 44, RG2003, 2006.

Bethke, C. M., and T. M. Johnson, Groundwater age and groundwater age dating, Annu. Rev. Earth Planet. Sci., 36, 121-152, 2008.

Cook, P., and D. K. Solomon, Recent advances in dating young groundwater: chlorofluorocarbons, 3h3he and 85 kr, Journal of hydrology, 191(1), 245-265, 1997.

Cook, P. G., and J.-K. Böhlke, Determining timescales for groundwater flow and solute transport, pp. 1-30, Springer, 2000.

Cornaton, F., and P. Perrochet, Groundwater age, life expectancy and transit time distributions in advective-dispersive systems: 1. generalized reservoir theory, Advances in Water Resources, 29(9), 1267-1291, doi:http://dx.doi.org/10.1016/j.advwatres.2005.10.009, 2006.

Cvetkovic, V., H. Cheng, and X.-H. Wen, Analysis of nonlinear effects on tracer migration in heterogeneous aquifers using Lagrangian travel time statistics, Water Resour. Res., 32(6), 1671-1680, 1996.

Cvetkovic, V., A. Fiori, and G. Dagan, Solute transport in aquifers of arbitrary variability: A time-domain random walk formulation, Water Resources Research, 50(7), 5759-5773, doi:10.1002/2014WR015449, cited By 6, 2014.

Cvetkovic, V. D., G. Dagan, and A. M. Shapiro, An exact solution of solute transport by onedimensional random velocity fields, Stochastic Hydrol. Hydraul., 5, 45-54, 1991.

Dagan, G., and E. Bressler, Solute dispersion in unsaturated soil at field scale, i, theory, Soil Sci. Soc. Am. J., 43, 461-466, 1979.

Dentz, M., and B. Berkowitz, Transport behavior of a passive solute in continuous time random walks and multirate mass transfer, Water Resour. Res., 39(5), 1111, 2003.

Dentz, M., A. Cortis, H. Scher, and B. Berkowitz, Time behavior of solute transport in heterogeneous media: transition from anomalous to normal transport, Adv. Water Resour., 27(2), 155-173, 2004.

Dentz, M., P. K. Kang, A. Comolli, T. Le Borgne, and D. R. Lester, Continuous time random walks for the evolution of Lagrangian velocities, Phys. Rev. Fluids, 1, 074,004, doi: 10.1103/PhysRevFluids.1.074004, 2016. 
Edery, Y., A. Guadagnini, H. Scher, and B. Berkowitz, Origins of anomalous transport in heterogeneous media: Structural and dynamic controls, Water Resour. Res., 50, doi:10.1002/2013WR015,111, doi:doi:10.1002/2013WR015111, 2014.

Engdahl, N. B., T. R. Ginn, and G. E. Fogg, Non-fickian dispersion of groundwater age, $W a-$ ter resources research, 48(7), 2012.

Favre, A.-C., S. El Adlouni, L. Perreault, N. Thiémonge, and B. Bobée, Multivariate hydrological frequency analysis using copulas, Water resources research, 40(1), 2004.

Ginn, T. R., On the distribution of multicomponent mixtures over generalized exposure time in subsurface flow and reactive transport: Theory and formulations for residence-timedependent sorption/desorption with memory, Water Resources Research, 36(10), 2885$2893,2000$.

Ginn, T. R., H. Haeri, A. Massoudieh, and L. Foglia, Notes on groundwater age in forward and inverse modeling, Transport in Porous Media, 79(1), 117-134, doi:10.1007/s11242009-9406-1, 2009.

Haslauer, C. P., P. Guthke, A. Bardossy, and E. A. Sudicky, Effects of non-gaussian copulabased hydraulic conductivity fields on macrodispersion, Water Resour. Res., 48, W07,507, doi:10.1029/2011WR011425, 2012.

Kang, P. K., M. Dentz, T. Le Borgne, and R. Juanes, Spatial markov model of anomalous transport through random lattice networks, Phys. Rev. Lett., 107, 180,602, 2011.

Kang, P. K., T. Le Borgne, M. Dentz, O. Bour, and R. Juanes, Impact of velocity correlation and distribution on transport in fractured media: field evidence and theoretical model, $\mathrm{Wa}$ ter Resour. Res., 51, 940-959, 2015.

Kreft, A., and A. Zuber, On the physical meaning of the dispersion equation and its solutions for different initial and boundary conditions, Chem. Eng. Sci., 33, 1471-1480, 1978.

Le Borgne, T., M. Dentz, and J. Carrera, A lagrangian statistical model for transport in highly heterogeneous velocity fields, Phys. Rev. Lett., 101, 090,601, 2008.

Leray, S., J.-R. de Dreuzy, O. Bour, T. Labasque, and L. Aquilina, Contribution of age data to the characterization of complex aquifers, Journal of Hydrology, 464, 54-68, 2012.

Leray, S., N. B. Engdahl, A. Massoudieh, E. Bresciani, and J. Mccallum, Residence time distributions for hydrologic systems: mechanistic foundations and steady-state analytical solutions, Journal of Hydrology, 543, 67-87, 2016.

Maloszewski, P., and A. Zuber, Principles and practice of calibration and validation of mathematical models for the interpretation of environmental tracer data in aquifers, Advances 
in Water Resources, 16(3), 173-190, 1993.

Massoudieh, A., and T. R. Ginn, The theoretical relation between unstable solutes and groundwater age, Water Resources Research, 47(10), 2011.

Massoudieh, A., S. Sharifi, and D. K. Solomon, Bayesian evaluation of groundwater age distribution using radioactive tracers and anthropogenic chemicals, Water Resources Research, 48(9), 2012.

Nelsen, R. B., An introduction to copulas, vol. 139, Springer Science \& Business Media, 2013.

Pope, S. B., Turbulent flows, 2001.

Salvadori, G., and C. De Michele, On the use of copulas in hydrology: theory and practice, Journal of Hydrologic Engineering, 12(4), 369-380, 2007.

Sklar, M., Fonctions de répartition à $n$ dimensions et leurs marges, Université Paris 8, 1959.

Solomon, D. K., D. P. Genereux, L. N. Plummer, and E. Busenberg, Testing mixing models of old and young groundwater in a tropical lowland rain forest with environmental tracers, Water resources research, 46(4), 2010.

Tyukhova, A., D. M., W. Kinzelbach, and M. Willmann, Mechanisms of anomalous dispersion in flow through heterogeneous porous media, Phys. Rev. Fluids, 1, 074,002, 2016.

Weissmann, G. S., Y. Zhang, E. M. LaBolle, and G. E. Fogg, Dispersion of groundwater age in an alluvial aquifer system, Water Resources Research, 38(10), 2002.

Woolfenden, L. R., and T. R. Ginn, Modeled ground water age distributions, Ground Water, 47(4), 547-557, 2009. 